\title{
Experiment Investigating the Connection between Weak Values and Contextuality
}

\author{
F. Piacentini, ${ }^{1}$ A. Avella, ${ }^{1}$ M. P. Levi, ${ }^{1}$ R. Lussana,${ }^{2}$ F. Villa,${ }^{2}$ A. Tosi, ${ }^{2}$ F. Zappa,${ }^{2}$ M. Gramegna, ${ }^{1}$ \\ G. Brida, ${ }^{1}$ I. P. Degiovanni, ${ }^{1}$ and M. Genovese ${ }^{1,3}$ \\ ${ }^{1}$ INRIM, Strada delle Cacce 91, I-10135 Torino, Italy \\ ${ }^{2}$ Politecnico di Milano, Dipartimento di Elettronica, Informazione e Bioingegneria, \\ Piazza Leonardo da Vinci 32, 20133 Milano, Italy \\ ${ }^{3}$ INFN, Via P. Giuria 1, I-10125 Torino, Italy \\ (Received 5 February 2016; published 2 May 2016)
}

\begin{abstract}
Weak value measurements have recently given rise to a great amount of interest in both the possibility of measurement amplification and the chance for further quantum mechanics foundations investigation. In particular, a question emerged about weak values being proof of the incompatibility between quantum mechanics and noncontextual hidden variables theories (NCHVTs). A test to provide a conclusive answer to this question was given by Pusey [Phys. Rev. Lett. 113, 200401 (2014)], where a theorem was derived showing the NCHVT incompatibility with the observation of anomalous weak values under specific conditions. In this Letter we realize this proposal, clearly pointing out the connection between weak values and the contextual nature of quantum mechanics.
\end{abstract}

DOI: 10.1103/PhysRevLett.116.180401

In 1988 Aharonov et al. introduced [1] weak value measurements [2-5], first realized in Refs. [6-13], that represent a new paradigm of quantum measurement where so little information is extracted from a single measurement that the state does not collapse.

Weak values, i.e., weak measurements of an operator performed on an ensemble of pre- and postselected states, present nonclassical properties, assuming anomalous values (i.e., values outside the eigenvalue range of the observable). In recent years they have been the subject of a great amount of interest for the possibility of amplifying the measurement of small parameters $[8,14-16]$ as well as for their nonclassical properties, allowing the investigation of fundamental aspects of quantum mechanics [3,4,17].

In particular, a question emerged about anomalous weak values constituting a proof of the incompatibility of quantum theory with noncontextual hidden variables theories (NCHVTs) [18-20], i.e., theories assuming that a predetermined result of a particular measurement does not depend on which other observables are simultaneously measured [31]. The possibility of testing this connection was recently unequivocally demonstrated in Ref. [21], showing that the mere observation of anomalous weak values is largely insufficient for this purpose, while noncontextuality is incompatible with the observation of anomalous weak values under specific experimental conditions. This result is of deep importance for understanding the role of contextuality in quantum mechanics, also in view of possible applications to quantum technologies.

To properly define the connection between noncontextuality and weak values, in Ref. [21] the following theorem was presented and proved, here in a form avoiding any (hidden) reference to quantum mechanics [22].
Theorem 1.-Let us suppose that we have a preparation procedure $\mathcal{P}_{\psi_{i}}$, a sharp measurement procedure $\mathcal{M}_{\psi_{f}}$ with outcomes PASS and FAIL, and a nondestructive measurement procedure $\mathcal{M}_{W}$ with outcomes $x \in \mathbb{R}$, such that

(1) The pre- and postselected states $\left|\psi_{i}\right\rangle$ and $\left|\psi_{f}\right\rangle$ are nonorthogonal, i.e.,

$$
p_{\psi_{f}}:=\mathbb{P}\left(\operatorname{PASS} \mid \mathcal{P}_{\psi_{i}}, \mathcal{M}_{\psi_{f}}\right)>0
$$

(2) Ignoring the postmeasurement state, $\mathcal{M}_{W}$ is equivalent to a two-outcome measurement with unbiased noise, i.e.,

$$
\begin{aligned}
\mathbb{P}\left(x \mid \mathcal{P}, \mathcal{M}_{W}\right)= & p_{n}(x-g) \mathbb{P}\left(1 \mid \mathcal{P}, \mathcal{M}_{\Pi}\right) \\
& +p_{n}(x) \mathbb{P}\left(0 \mid \mathcal{P}, \mathcal{M}_{\Pi}\right) \quad \forall \mathcal{P}
\end{aligned}
$$

for some sharp measurement procedure $\mathcal{M}_{\Pi}$ with outcomes 0 and 1 , and probability distribution $F(x)$ with median $x=0$.

(3) We can define a "probability of disturbance" $p_{d}$ such that, ignoring the outcome of $\mathcal{M}_{W}$, it affects the postselection in the same way as mixing it with another measurement:

$$
\begin{aligned}
\mathbb{P}\left(\operatorname{PASS} \mid \mathcal{P}, \mathcal{M}_{W}, \mathcal{M}_{\psi_{f}}\right)= & \left(1-p_{d}\right) \mathbb{P}\left(\operatorname{PASS} \mid \mathcal{P}, \mathcal{M}_{\psi_{f}}\right) \\
& +p_{d} \mathbb{P}\left(\operatorname{PASS} \mid \mathcal{P}, \mathcal{M}_{d}\right) \forall \mathcal{P}
\end{aligned}
$$

for some measurement procedure $\mathcal{M}_{d}$ with outcomes PASS and FAIL.

(4) The values of $x$ under the pre- and postselection have a negative bias that "outweighs" $p_{d}$; i.e., for the 
quantity $\quad p_{-}:=\left(p_{\psi_{f}}\right)^{-1} \int_{-\infty}^{0} \mathbb{P}\left(x, \operatorname{PASS} \mid \mathcal{P}_{\psi_{i}}, \mathcal{M}_{W}\right.$, $\left.\mathcal{M}_{\psi_{f}}\right) d x$ holds the inequality

$$
\mathcal{I}=p_{-}-\frac{1}{2}-\frac{p_{d}}{p_{\psi_{f}}}>0 .
$$

There is, then, no noncontextual ontological model for the preparation $\mathcal{P}_{\psi_{i}}$, measurement $\mathcal{M}_{W}$, and postselection on PASS of $\mathcal{M}_{\psi_{f}}$ satisfying the outcome determinism for sharp measurements.

Here, we present the very first experimental test of this theorem, performed by exploiting polarization weak measurements on heralded single photons [23,24].

In the framework of quantum mechanics, the preparation procedure $\mathcal{P}$ corresponds to the preselection of the polarization state $|\psi\rangle=\cos \theta|H\rangle+e^{i \beta} \sin \theta|V\rangle$ of our single photons, while the postselection process $\mathcal{M}_{\psi_{f}}$ is represented by the projector $\left|\psi_{f}\right\rangle\left\langle\psi_{f}\right|$, which yields for the probability $p_{\psi_{f}}$ the equivalence $p_{\psi_{f}}:=\mathbb{P}\left(\operatorname{PASS} \mid \mathcal{P}, \mathcal{M}_{\psi_{f}}\right)=$ $\left|\left\langle\psi_{f} \mid \psi\right\rangle\right|^{2}$. The nondestructive measurement procedure $\mathcal{M}_{W}$, instead, is implemented as a weak interaction induced by the unitary evolution $\hat{U}=\exp (-i g \hat{\Pi} \otimes \hat{P})$, with $g$ being the von Neumann coupling constant between the observable $\hat{\Pi}$ and a pointer observable $\hat{P}$ (see Fig. 1).

In our experiment, a single-photon state is prepared in the initial state $|\phi\rangle\rangle=|\psi\rangle \otimes\left|f_{x}\right\rangle$, with $\left|f_{x}\right\rangle=\int d x F(x)|x\rangle$, where $|F(x)|^{2}=p_{n}(x)$ is the probability density function of detecting the photon in the position $x$ of the transverse spatial plane. The shape of $p_{n}(x)$ is Gaussian with good approximation since the single photon guided in a singlemode optical fiber is collimated with a telescopic optical system (see Fig. 2), and by experimental evidence we can assume the (unperturbed) $p_{n}(x)$ to be centered around zero, with width $\sigma$.

The single photon undergoes a weak interaction realized as a spatial walk-off induced in a birefringent crystal,

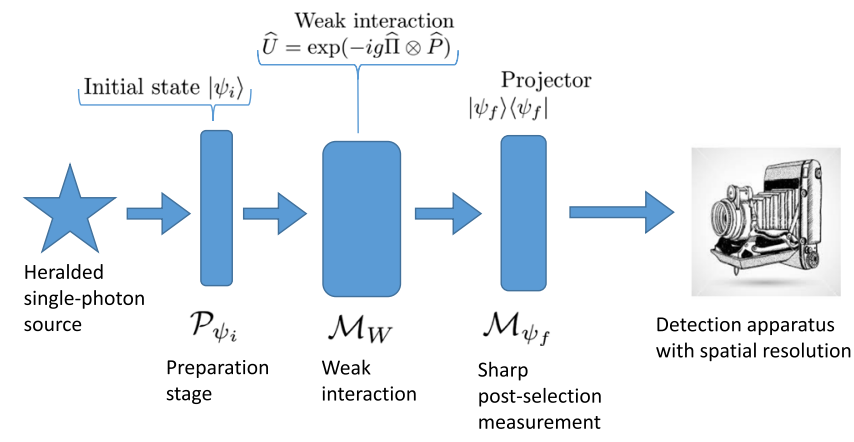

FIG. 1. Scheme of a "gedanken" experiment for the noncontextuality test of Theorem 1. The single photons, prepared in the initial state $\left|\psi_{i}\right\rangle$, undergo a weak interaction and a sharp postselection measurement before being addressed to a detector with spatial resolution. For each part of the scheme, both the noncontextual (below) and quantum mechanical (above) description of its effect are reported. described by the unitary transformation $\hat{U}$. The probability of finding the single photon in the position $x_{0}$ of the transverse plane [see Eq. (2)] can be evaluated as

$$
\mathbb{P}\left(x_{0} \mid \mathcal{P}, \mathcal{M}_{W}\right)=\operatorname{tr}\left[M_{x_{0}}|\psi\rangle\langle\psi| M_{x_{0}}^{\dagger}\right],
$$

where $\left.M_{x_{0}}|\psi\rangle=\left\langle x_{0}|\hat{U}| \phi\right\rangle\right\rangle$. The quantities $\mathbb{P}\left(1 \mid \mathcal{P}, \mathcal{M}_{\Pi}\right)$ and $\mathbb{P}\left(0 \mid \mathcal{P}, \mathcal{M}_{\Pi}\right)$ in Eq. (2) correspond, respectively, to the probability that the single photon undergoes or does not the weak interaction in the crystal, i.e., $\mathbb{P}\left(1 \mid \mathcal{P}, \mathcal{M}_{\Pi}\right)=$ $\langle\psi|\hat{\Pi}| \psi\rangle \quad$ and $\mathbb{P}\left(0 \mid \mathcal{P}, \mathcal{M}_{\Pi}\right)=1-\mathbb{P}\left(1 \mid \mathcal{P}, \mathcal{M}_{\Pi}\right)=\langle\psi|\hat{\tilde{\Pi}}| \psi\rangle$ (with $\hat{\tilde{\Pi}}=I-\hat{\Pi})$.

The quantity $\mathbb{P}\left(\operatorname{PASS} \mid \mathcal{P}, \mathcal{M}_{d}\right)$ in Eq. (3) represents an unknown measurement process, but what we need to demonstrate is just that its contribution is negligible because of the nondestructive nature of the measurement $\mathcal{M}_{W}$ (since we exploited the weak measurement paradigm). The parameter $p_{d}$, quantifying such a contribution (i.e., the disturbance that $\mathcal{M}_{W}$ causes to the subsequent sharp measurement $\mathcal{M}_{\psi_{f}}$ ) can be evaluated as the amount of decoherence induced on the single photon by the weak interaction $\hat{U}, p_{d}=1-e^{-\left(g^{2} / 4 \sigma^{2}\right)}$.

Our experimental setup (Fig. 2) consists of a $796 \mathrm{~nm}$ mode-locked Ti:sapphire laser (repetition rate, $76 \mathrm{MHz}$ ) whose second harmonic emission pumps a $10 \times 10 \times$ $5 \mathrm{~mm} \mathrm{LiIO}_{3}$ nonlinear crystal, producing type-I parametric down-conversion. The idler photon $\left(\lambda_{i}=920 \mathrm{~nm}\right)$ is coupled to a single-mode fiber (SMF) and then addressed to a silicon single-photon avalanche diode (SPAD), heralding the presence of the correlated signal photon

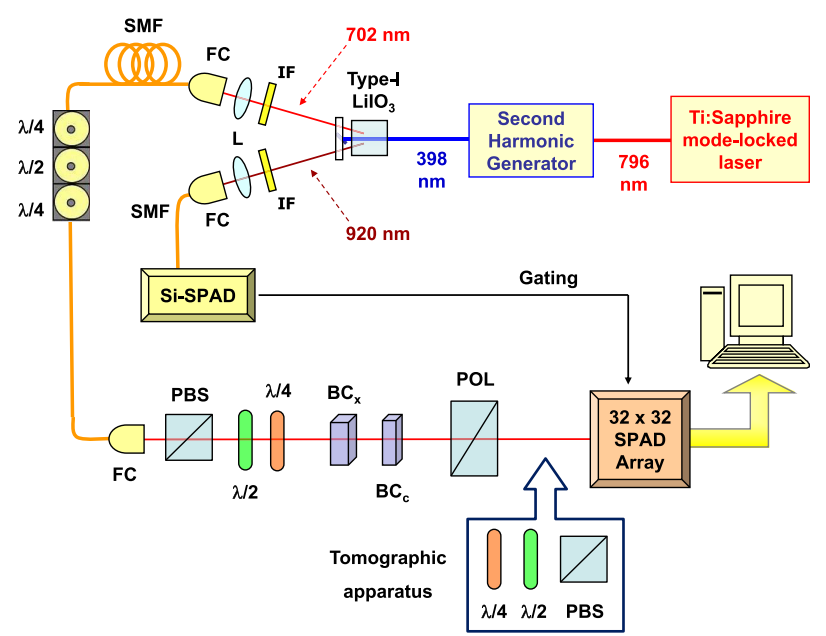

FIG. 2. Experimental setup. After the weak interaction with a birefringent crystal, the heralded single photon is projected onto the postselected state by a Glan linear polarizer, then addressed to the space-resolving detector. For some consistency checks, a tomographic apparatus can be inserted at some point between the polarizer and the detector. SHG, second harmonic generator; PBS, polarizing beam splitter; BC, birefringent crystal; POL, Glan polarizer. 
$\left(\lambda_{s}=702 \mathrm{~nm}\right)$ that, after being SMF coupled, is sent to a launcher and then to the free-space optical path where the weak value evaluation is performed.

We have estimated the quality of our single-photon emission, obtaining a $g^{(2)}(0)$ value (or, more properly, a parameter $\alpha$ value [25-27]) of $0.13 \pm 0.01$ without any background or dark-count subtraction.

After the launcher, the heralded single photon state is collimated by a telescopic system, and then prepared (preselected) in the chosen state $\left|\psi_{i}\right\rangle$ by means of a calcite polarizer followed by a quarter-wave plate and a half-wave plate. The weak measurement is carried out by a $1 \mathrm{~mm}$ long birefringent crystal $\left(\mathrm{BC}_{x}\right)$, whose extraordinary $(e)$ optical axis lies in the $X-Z$ plane, with an angle of $\pi / 4$ with respect to the $Z$ direction. Because of the spatial walk-off experienced by the vertically polarized photons, horizontal- and vertical-polarization paths get slightly separated along the $X$ direction, inducing in the initial state $\left|\psi_{i}\right\rangle$ a small decoherence (below 1\%) that keeps it substantially unaffected. Subsequently, the birefringent crystal $\mathrm{BC}_{c}$ performs a phase compensation that is tuned in order to nullify the temporal walk-off generated in $\mathrm{BC}_{x}$. From the parameters $g$ and $\sigma$ of our system, we estimated $p_{d}=0.0019 \pm 0.0002$.

After the weak measurement is performed, the photon meets a Glan polarizer projecting it onto the postselected state $\left|\psi_{f}\right\rangle$. Then, the photon goes to the detection device, a two-dimensional array made of $32 \times 32$ "smart pixels," fabricated in a cost-effective $0.35 \mu \mathrm{m}$ standard complementary metal-oxide semiconductor technology. Each pixel hosts a $30 \mu \mathrm{m}$ diameter silicon SPAD detector with $15 \%$ photon detection efficiency (PDE) at $702 \mathrm{~nm}$ (the peak PDE is $55 \%$ at $420 \mathrm{~nm}$ ), and its front-end electronics for sensing and quenching the avalanche and counting the number of detected photons [28]. The SPADs are gated with $6 \mathrm{~ns}$ integration windows, triggered by the SPAD detector of the heralding arm; spurious detections within such integration windows are minimized thanks to the array's excellent dark-counting rate performance (120 cps at room temperature, with just 3\% hot pixels).

A removable polarization tomographic apparatus [29,30] is inserted between the Glan polarizer and the detector only when needed, i.e., to verify the fulfillment of the condition in Eq. (3).

Figure 3 shows the plot of the quantity $\mathcal{I}$ of Eq. (4) with respect to the angle $\theta$ of the linearly polarized postselection state $\left|\psi_{f}\right\rangle=\cos (\theta)|H\rangle+\sin (\theta)|V\rangle$, with $\left|\psi_{i}\right\rangle=(1 / \sqrt{2})$ $(|H\rangle-|V\rangle)$. Experimentally, by choosing $\theta=0.18 \pi$ we obtained the value $\mathcal{I}^{(\exp )}=0.063 \pm 0.011$, in excellent agreement with the quantum-mechanical predictions and 5.7 standard deviations distant from the noncontextual bound.

In order to demonstrate the validity of Eq. (2), we removed the polarizer realizing $\mathcal{M}_{\psi_{f}}$, so that we could estimate the probability $Q(x)$ that a single photon prepared in any arbitrary polarization state $|\psi\rangle$ is detected at the

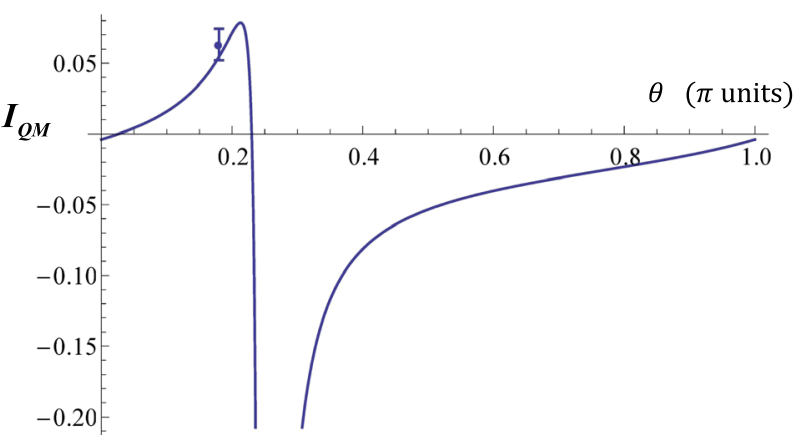

FIG. 3. $\mathcal{I}$ plot, with respect to the postselection angle $\theta$. For $\theta=0.18 \pi$, we obtained $\mathcal{I}^{(\exp )}=0.063 \pm 0.011$, certifying a violation of the noncontextual bound of 5.7 standard deviations.

position $x$ after the weak interaction, a faithful estimation of $\mathbb{P}\left(x \mid \mathcal{P}, \mathcal{M}_{W}\right)$. This task was accomplished by sending the (tomographically complete) set of four different input states $\{|H\rangle, \quad|V\rangle, \quad|+\rangle=(1 / \sqrt{2})(|H\rangle+|V\rangle), \quad|R\rangle=(1 / \sqrt{2})$ $(|H\rangle-i|V\rangle)\}$, and measuring $Q(x)$ in the absence of the polarizer performing the state postselection. Then, we compared the measured $Q(x)$ with the expected one obtained from the right side of Eq. (2); the function $p_{n}(x)$ is reconstructed by fitting the spatial profile in the absence of the weak interaction $(\mathbb{P}(1 \mid \mathcal{P}, \hat{\Pi})=0)$, and the value of $g$ is estimated by maximizing the interaction $(\mathbb{P}(0 \mid \mathcal{P}, \hat{\Pi})=0)$.

The validity of our approach is shown by the fidelity between the measured $Q(x)$ and the expected one, $Q^{(e)}(x)$, evaluated by sampling more than 230 points in the region where $Q(x)$ is significantly nonzero, obtaining 0.997 , $0.991,0.994,0.996$ for the four input states $|H\rangle,|V\rangle$, $|+\rangle$, and $|R\rangle$, respectively. To confirm the quality of our reconstruction, we also performed a pixel-by-pixel proximity test of the two probability distributions for the pixels where the $Q(x)$ is significantly nonzero. We define the proximity between the two distributions as

$$
\operatorname{PROX}_{\psi}(x)=\left[\frac{2 Q(x) Q^{(e)}(x)}{[Q(x)]^{2}+\left[Q^{(e)}(x)\right]^{2}}\right]^{\frac{1}{2}} .
$$

As shown in Fig. 4, for all of the input states the proximity between the two distributions is larger than 0.99 for almost every point, demonstrating that our experimental setup provides a faithful realization of the condition in Eq. (2).

Finally, to prove that the condition of Eq. (3) is fulfilled, we used the following method, based on the comparison between experimental probabilities collected in different conditions, in order to get rid of any possible bias due to quantum-mechanical assumptions. First, we prepared a (tomographically complete) set of states and registered the detection probabilities $P$ and $\tilde{P}$, obtained with the Glan polarizer projecting the single-photon states onto $\left|\psi_{f}\right\rangle$ and its orthogonal $\left|\tilde{\psi}_{f}\right\rangle$. For each input state $|\psi\rangle$, these 

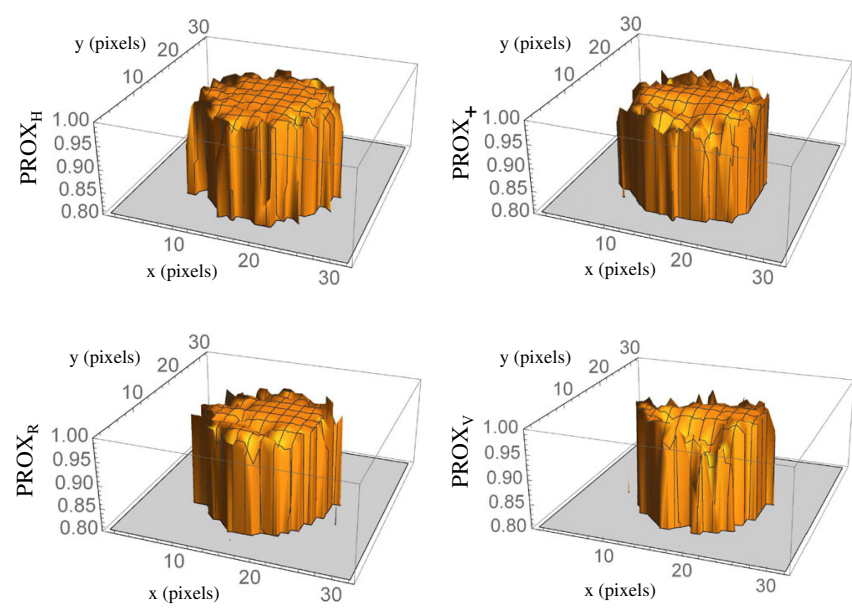

FIG. 4. $\operatorname{PROX}_{\psi}(x)$ evaluated for the input states $|\psi\rangle=|H\rangle$, $|+\rangle,|R\rangle$, and $|V\rangle$. For all of them, the proximity is above 0.99 for almost all of the pixels, a clear sign that the condition in Eq. (2) is satisfied.

probabilities are given by the photon counts divided by the trigger counts $N_{T}$ of the heralded single-photon source $\left(P=\left(N^{\psi_{f}} / N_{T}\right), \tilde{P}=\left(N^{\tilde{\psi}_{f}} / N_{T}\right)\right)$. Second, we switched the position of the preparation stage and the birefringent crystals, in order to nullify the weak interaction without altering the optical losses in the system, and performed the same set of acquisitions. To get rid of any bias, proper dark counts and background noise subtraction is performed.

For each input state, these two acquisitions correspond, respectively, to the evaluation of the quantities $\mathbb{P}\left(\mathrm{PASS} \mid \mathcal{P}, \mathcal{M}_{W}, \mathcal{M}_{\psi_{f}}\right)$ and $\mathbb{P}\left(\mathrm{PASS} \mid \mathcal{P}, \mathcal{M}_{\psi_{f}}\right)$ reported in Eq. (3). Concerning the third one, connected to the unknown measurement procedure $\mathcal{M}_{d}$, one can notice that by definition $\mathbb{P}\left(\mathrm{PASS} \mid \mathcal{P}, \mathcal{M}_{d}\right) \in[0,1]$, and thus one can write

$$
\begin{aligned}
& \left(1-p_{d}\right) \mathbb{P}\left(\operatorname{PASS} \mid \mathcal{P}, \mathcal{M}_{\psi_{f}}\right) \\
& \quad \leq \mathbb{P}\left(\operatorname{PASS} \mid \mathcal{P}, \mathcal{M}_{W}, \mathcal{M}_{\psi_{f}}\right) \\
& \quad \leq\left(1-p_{d}\right) \mathbb{P}\left(\operatorname{PASS} \mid \mathcal{P}, \mathcal{M}_{\psi_{f}}\right)+p_{d}
\end{aligned}
$$

giving an upper and a lower bound to the parameter $p_{d}$. The collected data allowed us to obtain $(0.000021 \pm 0.000014) \leq p_{d} \leq(0.086 \pm 0.050)$; the $p_{d}$ value derived by the system parameters fits perfectly in this range.

As a further consistency check, we tested the output state after the sharp measurement $\mathcal{M}_{\psi_{f}}$ (realized by the Glan polarizer) by inserting the tomographic apparatus in the setup (see Fig. 2), implicitly accepting some quantummechanical assumptions. Such an apparatus was exploited to perform two different experiments.

In the first one, we used it to project the state after $\mathcal{M}_{\psi_{f}}$ onto $\psi_{f}$ and $\tilde{\psi}_{f}$. While we were able to detect a clear signal with the tomographic device realizing the same projection as the Glan polarizer (i.e., onto $\psi_{f}$ ), the amount of signal registered with the tomographer projecting onto $\tilde{\psi}_{f}$ was so small as to be completely indistinguishable from the detector noise, as expected when photons undergo two subsequent projections onto orthogonal axes. This confirms that the sharp measurement process $\mathcal{M}_{\psi_{f}}$ is performing a projection onto the state $\psi_{f}$.

In the second experiment, we instead performed the tomographic reconstruction of the state after the postselection on $\left|\psi_{f}\right\rangle$. We prepared a tomographically complete set of input states, i.e., $|H\rangle,|+\rangle,|L\rangle=(1 / \sqrt{2})(|H\rangle+$ $i|V\rangle)$, and $|R\rangle$, and we tried to reconstruct via quantum tomography the state after the $\mathcal{M}_{\psi_{f}}$ measurement process. From the tomographic reconstructions, we obtained states whose fidelities with respect to the chosen $\left|\psi_{f}\right\rangle$ were $\mathcal{F}_{H}=0.9995, \mathcal{F}_{+}=0.9999, \mathcal{F}_{L}=0.9991$, and $\mathcal{F}_{R}=$ 0.9811 . These values led us to estimate $p_{d}=0.0051 \pm$ 0.0046 , fitting the range obtained for $p_{d}$ with the method presented above, and in good agreement with the $p_{d}$ value derived from the system experimental parameters $\left(p_{d}=0.0019 \pm 0.0002\right)$.

Since all conditions of the theorem presented in Ref. [21] have been verified, we can assess that the results of our experiment clearly violate the noncontextual bound for the quantity $\mathcal{I}$ in Eq. (4), providing a sound demonstration of the connection between weak values and the intrinsic contextual nature of quantum mechanics.

This work has been supported by EMPIR-14IND05 "MIQC2" (the EMPIR initiative is cofunded by the EU H2020 and the EMPIR Participating States), and by the John Templeton Foundation (Grant No. 43467). We are deeply indebted to Matthew Pusey for the fruitful discussions and theoretical support.

[1] Y. Aharonov, D. Z. Albert, and L. Vaidman, Phys. Rev. Lett. 60, 1351 (1988).

[2] A. G. Kofman, S. Ashhab, and F. Nori, Phys. Rep. 520, 43 (2012).

[3] J. Dressel, M. Malik, F. M. Miatto, A. N. Jordan, and R. W. Boyd, Rev. Mod. Phys. 86, 307 (2014).

[4] B. Tamir and E. Cohen, Quanta 2, 7 (2013).

[5] Y. Aharonov, E. Cohen, and A. C. Elitzur, Phys. Rev. A 89, 052105 (2014).

[6] N. W. M. Ritchie, J. G. Story, and R. G. Hulet, Phys. Rev. Lett. 66, 1107 (1991).

[7] G. J. Pryde, J. L. O’Brien, A. G. White, T. C. Ralph, and H. M. Wiseman, Phys. Rev. Lett. 94, 220405 (2005).

[8] O. Hosten and P. Kwiat, Science 319, 787 (2008).

[9] M. E. Goggin, M. P. Almeida, M. Barbieri, B. P. Lanyon, J. L. O'Brien, A. G. White, and G. J. Pryde, Proc. Natl. Acad. Sci. U.S.A. 108, 1256 (2011).

[10] J. Dressel, C. J. Broadbent, J. C. Howell, and A. N. Jordan, Phys. Rev. Lett. 106, 040402 (2011). 
[11] J. P. Groen, D. Ristè, L. Tornberg, J. Cramer, P. C. de Groot, T. Picot, G. Johansson, and L. DiCarlo, Phys. Rev. Lett. 111, 090506 (2013).

[12] S. Sponar, T. Denkmayr, H. Geppert, H. Lemmel, A. Matzkin, J. Tollaksen, and Y. Hasegawa, Phys. Rev. A 92, 062121 (2015).

[13] T. C. White et al., NPJ Quantum Inf. 2, 15022 (2016).

[14] K. J. Resch, Science 319, 733 (2008).

[15] J. Z. Salvail, M. Agnew, A. S. Johnson, E. Bolduc, J. Leach, and R. W. Boyd, Nat. Photonics 7, 316 (2013).

[16] J. S. Lundeen, B. Sutherland, A. Patel, C. Stewart, and C. Bamber, Nature (London) 474, 188 (2011).

[17] F. Piacentini et al., arXiv:1508.03220.

[18] R. W. Spekkens, Phys. Rev. A 71, 052108 (2005).

[19] J. Tollaksen, J. Phys. A 40, 9033 (2007).

[20] M. Genovese, Phys. Rep. 413, 319 (2005).

[21] M. Pusey, Phys. Rev. Lett. 113, 200401 (2014).

[22] M. Pusey (private communication).

[23] M. D. Eisaman, J. Fan, A. Migdall, and S. V. Polyakov, Rev. Sci. Instrum. 82, 071101 (2011).
[24] G. Brida, I. P. Degiovanni, M. Genovese, A. Migdall, F. Piacentini, S. V. Polyakov, and I. Ruo Berchera, Opt. Express 19, 1484 (2011).

[25] P. Grangier, G. Roger, and A. Aspect, Europhys. Lett. 1, 173 (1986).

[26] G. Brida et al., Appl. Phys. Lett. 101, 221112 (2012).

[27] G. Brida, M. Genovese, M. Gramegna, and E. Predazzi, Phys. Lett. A 328, 313 (2004).

[28] F. Villa et al., IEEE J. Sel. Top. Quantum Electron. 20, 364 (2014).

[29] D. F. V. James, P. G. Kwiat, W. J. Munro, and A. G. White, Phys. Rev. A 64, 052312 (2001).

[30] Y. I. Bogdanov, G. Brida, M. Genovese, S. P. Kulik, E. V. Moreva, and A. P. Shurupov, Phys. Rev. Lett. 105, 010404 (2010).

[31] NCHVTs are a subset of focal realistic hidden variables theories, assuming that a predetermined result of a particular measurement does not depend on which other observables are simultaneously measured in a causally nonconnected region; see M. Genovese, Phys. Rep. 413, 319 (2005). 